\title{
FocSge1 in Fusarium oxysporum f. sp. cubense race 1 is essential for full virulence
}

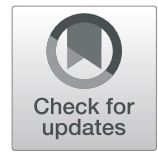

Vartika Gurdaswani', Siddhesh B. Ghag ${ }^{1 *}$ (D) and Thumballi R. Ganapathi ${ }^{2}$

\begin{abstract}
Background: Fusarium wilt disease of banana is one of the most devastating diseases and was responsible for destroying banana plantations in the late nineteenth century. Fusarium oxysporum f. sp. cubense is the causative agent. Presently, both race 1 and 4 strains of Foc are creating havoc in the major banana-growing regions of the world. There is an urgent need to devise strategies to control this disease; that is possible only after a thorough understanding of the molecular basis of this disease.

Results: There are a few regulators of Foc pathogenicity which are triggered during this infection, among which Sge1 (Six Gene Expression 1) regulates the expression of effector genes. The protein sequence is conserved in both race 1 and 4 strains of Foc indicating that this gene is vital for pathogenesis. The deletion mutant, FocSge1 displayed poor conidial count, loss of hydrophobicity, reduced pigmentation, decrease in fusaric acid production and pathogenicity as compared to the wild-type and genetically complemented strain. Furthermore, the C-terminal domain of FocSge1 protein is crucial for its activity as deletion of this region results in a knockout-like phenotype.

Conclusion: These results indicated that FocSge1 plays a critical role in normal growth and pathogenicity with the C-terminal domain being crucial for its activity.
\end{abstract}

Keywords: Fusarium oxysporum f. sp. cubense, Banana, Fusarium wilt, Virulence, FocSge1, Transcription factor, SIX1

\section{Background}

Banana (Musa spp.) is one of the important staple food crops of the world. However, the production is constrained largely due to diseases including the Fusarium wilt disease caused by Fusarium oxysporum f. sp. cubense (Foc). Physiologically, Foc is divided into four races, of which race 1 and Tropical race 4 (TR4) are important. Foc race 1 caused havoc in the late nineteenth century resulting in the shifting of the banana industry from race 1-susceptible Gros Michel variety to the race 1-resistant Cavendish variety [1]. However, the recently evolved TR4 strain infects almost all edible cultivars of banana including the race 1-resistant Cavendish; and thus far, no known

\footnotetext{
*Correspondence: siddhesh.ghag@cbs.ac.in

${ }^{1}$ School of Biological Sciences, UM-DAE Centre for Excellence in Basic

Sciences, University of Mumbai campus, Kalina, Santacruz (E), Mumbai 400 098, India

Full list of author information is available at the end of the article
}

substitute for Cavendish exists. And, currently no known effective strategies to curb this pathogen have been offered from any quarters (industry or research).

In the absence of its host (banana plant) Foc remains in the soil as a saprophyte or survives as dormant chlamydospores [2]. In the presence of banana roots, Foc switches to the pathogenic mode and colonizes it. During this switch, there is a complete reprogramming of the expression of genes required for pathogenicity such as cell wall-degrading enzymes, effector proteins and toxic secondary metabolites. However, this transition phase is governed by a few master transcriptional regulators which further control the gene expression profile. A similar morphological switching was found to be controlled by WOR1 from a white to opaque cell type in Candida albicans and RYP1 from filamentous to a yeast form in Histoplasma capsulatum. Wor1 orthologs have been identified in phytopathogenic fungi that play a 
crucial role in pathogenicity. Fusarium graminearum Fgp1 and Botrytis cinerea Reg1 genes regulate pathogenicity and trichothecene toxin synthesis [3, 4]. Sge1, a homolog of Worl was first identified in Fusarium oxysporum f. sp. lycopercisi (Fol) and was shown to be required for parasitic growth and virulence in tomato [5]. Deletion or inactivation of SGE1 resulted in reduced pathogenicity and was further correlated with the reduced levels of toxin production and effector proteins.

SGE1 from Foc TR4 was shown to regulate a series of genes required for infection. An SGE1 deletion mutant of Foc TR4 demonstrated impaired conidiation and complete loss of virulence on banana plantlets [6]. However, Sge1 deletion mutant of Fol showed no effect on colonization capability, whereas colonization was compromised in Sge1 mutant of Foc. A homolog of Sge1 in Fusarium verticillioides (SGE1) is required for pathogenicity and synthesis of secondary metabolites such as fumonisins and fusarins [7]. Nonetheless, SGE1 in $F$. verticillioides was not essential for conidiation as it was in Foc and Fol. This indicates that SGE1 has multiple yet different roles in different phytopathogenic fungi. Sge1 is known to regulate the expression of major effector genes called SIX (secreted in xylem) which are synthesized and secreted into the xylem of the infected tomato plant [5]. Among the SIX proteins, SIX1 is most crucial as its deletion led to reduced virulence of Foc TR4 on banana plants [8], F. oxysporum f. sp. conglutinans on cabbage [9] and F. oxysporum f. sp. lycopersici on tomato [10]. Furthermore, even though SIX proteins are homologous, they are host-selective and inefficient to complement in other pathotypes [9]. This clearly indicates that the interaction of the host plant with the pathogenic strain is differential and intricate. Thus, deciphering the virulence in each pathosystem is critical for a thorough understanding of the infection paradigm.

Pathogen infection strategies and molecular pathways in pathogenesis have always been a subject of rigorous exploration and research. Newer perceptions in this direction will further help in developing appropriate disease management strategies. Depending on the previously described variable, but critical roles of Sge1 homologs in pathogenicity and development, we sought to investigate the role of FocSge1 in Foc race 1. Our results demonstrate that FocSge1 is required for conidiation, pigmentation, colony hydrophobicity and full virulence on banana plants.

\section{Results}

Identification of FocSge1 in F. oxysporum f. sp. cubense

FocSge1 gene was PCR amplified from the genomic DNA of Foc race 1 and sequenced. The coding sequence of FocSge1 was translated in silico to its corresponding amino acid sequence using ExPasy translate tool and used for multiple sequence alignment with its closest homologs. FocSge1 is a 330 aa protein with a theoretical pI of 8.47. It has a Gti-Pac2 domain at the N-terminal end. The Gti1 protein in Schizosaccharomyces pombe helps in utilization of gluconate under glucose starvation condition whereas Pac2 protein controls the sexual development in a pathway independent of the cAMP cascade. A multiple sequence alignment of FocSge1 with hypothetical proteins containing Gti-Pac2 domain from Foc race 1 (ENH74652), race 4 (EMT70718), related to S. pombe pac2 protein from Fusarium fujikuroi (SCN84561), Gti1/Pac2 family protein from Colletotrichum tofieldiae (KZL63430) and cAMP-independent regulatory protein from Colletotrichum orbiculare (ENH87589) showed a high level similarity (Fig. 1). Gti/Pac2 domain is also shared by some crucial transcription factors involved in dimorphic switching. A multiple sequence alignment of FocSge1 protein and gluconate transporter inducer Gtil from S. pombe (NP_592911) was performed with Bcreg1 from Botrytis cinerea (XP_024547925), Ryp1 from Histoplasma capsulatum (ABX74945.1) and Wor1p from Candida albicans SC5314 (AOW26645.1) (Supplementary Figure S1). These sequences were found to be conserved at the N-terminal end (1-120 amino acid residues). The two motifs KRWTD and WSPSR were highly conserved in all the five sequences analyzed. This indicates that FocSge1 is an important protein involved in switching of lifestyles, probably from saprophytic to parasitic mode in Foc race1. Being crucial, the protein could be conserved in most of the Fusarium oxysporum strains. Hence, a multiple sequence alignment of protein sequences from Fusarium oxysporum f. sp. lycopersici 4287 (XP_018248224), Fusarium sp. FOSC 3-a (EWY91661), Fusarium oxysporum f. sp. pisi HDV247 (EXA45922), Fusarium oxysporum Fo5176 (EGU84598) and Fusarium oxysporum Fo47 (EWZ38328) was performed. The Sge1 homologs in these strains are identical except for 6 mismatches among them and an additional proline-glutamine residues in Fo47 strain at 306307 position in the primary sequence (Supplementary Figure S2).

The $2 \mathrm{kbp}$ upstream sequence of FocSge1 was analyzed for the presence of GATA element. GATA factors bind to a conserved signature element WGATAR (W $=\mathrm{T}$ or $\mathrm{A}$ and $\mathrm{R}=\mathrm{G}$ or $\mathrm{A}$ ) present in the regulatory region of the gene. In FocSge1 upstream sequence we found one GATA element with sequence AGATAG (Fig. 2). GATA factors are regulated by the type of nitrogen source in the medium which in turn control the expression of genes that are present downstream of this element. Since GATA element was identified in FocSge1 upstream region, it is likely that the expression of FocSge1 is indirectly regulated by the nitrogen sources.

Targeted deletion of FocSge1 gene and complementation To investigate the function of FocSge1, pCSN44- $\triangle$ FocSge1 deletion vector was transformed into Foc race 1 


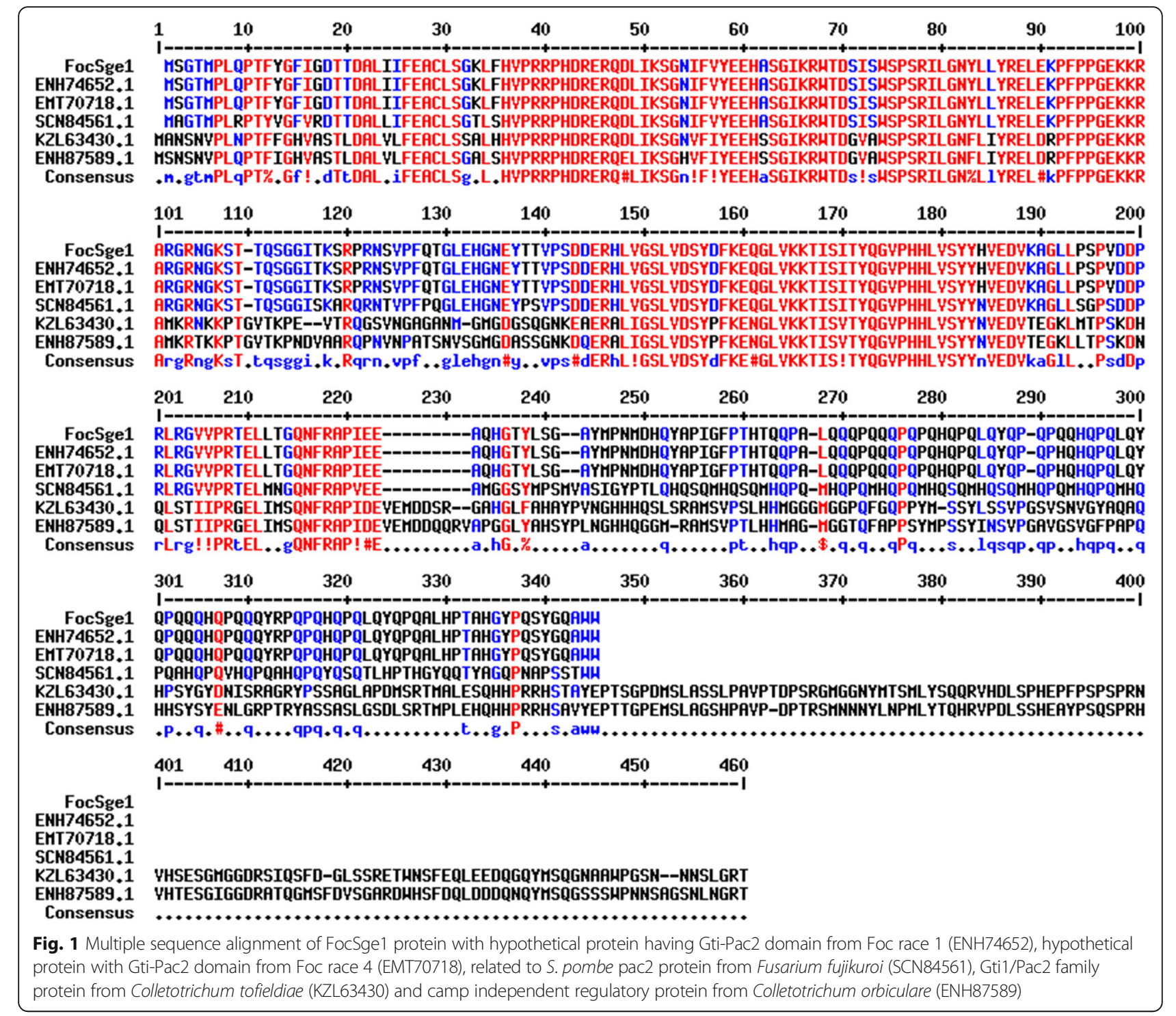

spheroplasts and transformants obtained through homologous recombination were selected on hygromycincontaining medium (Fig. 3a). First fifteen transformants obtained were screened for the absence of FocSge1 coding sequence by PCR using FocSge1cds Fw / FocSge1cds Rv primer set (Fig. 3c). Two transformants K09 and K12 were confirmed knockout FocSge1 transformants. These transformants were further confirmed for the presence of the hygromycin coding sequence for an amplicons of $1024 \mathrm{bp}$ using PCR (Fig. 3d). Among the two transformants, K09 was selected for further analysis. The K09 deletion mutant was complemented with the full-length FocSge1 gene or its truncated version and the transformants were selected on phleomycin. Of the several transformants obtained, one full-length (C7) and one truncated mutant (T10) were selected for further analysis. The complemented mutant strains were screened for the presence of the FocSge1 coding sequence by PCR. An amplified fragment of $993 \mathrm{bp}$ in full-length complementation strain comparable with the wild-type and $780 \mathrm{bp}$ in truncated mutant was observed that was completely absent in the K09 deletion mutant strain (Fig. 3c).

Relative expression analysis of FocSge 1

To check the expression of FocSge1 gene in the wildtype and mutant strains, real-time PCR (RT-PCR) was carried out. Cell suspension cultures of banana CV. Rasthali inoculated with conidia were assessed after $60 \mathrm{~h}$ for the expression. The expression of FocSge1 was induced in wild-type strain infected banana cells whereas no expression was seen in the deletion stain K09. However, the expression was restored in the complementation and truncated strain, C7 and T10 respectively (Fig. 3e). 


\begin{abstract}
GATGCATAAGGAGGATGAAGGGAGGAGGGGTGCAGGGGGTGGAGGACGTGTCCCCAGGCGTGTACCTATCAAAGCCAGATCCACCTC CAAAGCACTTCTCATGAGGAGAGGTACAGCCTTCAGGGACGAGGTGAGGTAGTTCCAGCTGGGATGGGACGGGGGATGGGGATGTAG GTTTTCATTGCATTAAGCTTCCCGTCGATTTGGAGAAGGGCTGAACAGCTCTAACTAGATCTGAATCTTAGAATTTAGATAGTGAAGAG CTTCTTTTGAATTGAAGAAACCATCTTGAGAGAATGATTCGCCCAGGCCCTTGCTCAAATAGAGATCGAATTCTTCCAGAGTGAGACAC AGATCCGACCTGGAGGGAACACCAACATGATGGCAGGTACCTGACCTCGACACAGGTCGCGGTCTCTCGGCCGCATACAAACAGCCCG CAACTACCTAGAGTAGATCCGGACAGCCACCACCCACCTCAACAGCAGGTGTGAGACACCACTACAAGCACGTACCGATACGGTAGGT AGGCTTTTGAGGTACTTCGGCACATGCTCCGACAGCGTGATGAAGGGGCTTTGCTGGGGTATCTGTCCAGTCAGCGGAAGTACCTGCCC AATGGTGATATCAGGATGTTTCCAATGGGCCTGACCAGACGAGGAGTCTGTGAGGAGGCAGGCAGGCGGGCGGGCGGGTACGTACCTT CCCCATACCTTGCCGTACATCTTTTTGCTGCCCTACCAACTCAATACCACCTGTATGGACTGCCGTACTGACTGACCCGACCTATCCCCA ATATCCCTGGAGAGAGCTGGCCGCGGGTACCGTACCGTACCGTAGGGAGGTTAGGTACCTGCTTGCAGTTCCATTTCCCTCCTCCTCCTT CCTTAGGAGGTCCAAACGACATGGATGGCAATGACAATGTGAATGACACGCGTGGATATGGAACGGCATCTAAGCTGATGGAAAGGCC GGATACAGACACTACCCAGGATTTGGTTGACGCAGACGGGAGCCAGCCAACAAGTGCCAGGCCAGCCAGGCCCCAGATTCAAGCCCAG GGAATTACTAGGTACCTACCTGCTCTTTCCTAAATCCAAGCTTGAGTCCATCCATCTATGCCATGGAAGCGCAAGTGCAACTAGCCTTTA GCCTGCCCTGTAGTGTAGATTGCCTACTTGGGTAAGTGCATTGCATTGCATTCGACTACATTACATCCAAGCCATCCACTTACACCTCAA CTTCACTTCACCACATTTCATCCACATTATTCTCTTGTTCTTTATAATTTTCTTCTTCTTCTTGTTGACCATCCATTGTTTACTTTCACCAAC TGGCTTTTACAATCATTGCCACTTAACGCTTGAACGAAATACGCACACACCTCGAACCAATTACTTCGTGCCCTCACTTGCTCAGAGATT CTTCACCCAGAAGTCAATGGTGAAATAAAGAAACAGCTCACGCTCTCCTATACGCCTACCCGCCCGCCACAACTGGAATACTTTCTTCC TTGAAAATAGCCTTTTTACAAAGGCCCTCGTGATACCCATTTGCACGCACTGTTTGGTGCGGTTTACTTTGTTGAGTTCGAGAGCTCAAC TTTCGCCATTGTTCGGTGCGGTAGCTCTCGCGATTAGAGAGAAGCGCCTATCAACATATGCAGCTGAATTGACGATTCGAACAAGCCCT ACGATTACTCCCATCTTATTTGTCACAGCAACCTGTTGAAAGGCAGTGTTCGCCTCATGAGGATTTTTGGGAGCAACACAGCGCGGCCC ATATTCCTATAAATACTGAGGCCATCGTCGATTTTAGACTTGAAGTTTTCTCCCCCAAAGTCAACCTCTTGAGATCACTGAGATTATTTT CCСCATTTCCTCGAGACTGACTTCGAGTCCAAGGTTAAGATCACTTACACTTGCACTCACTCTACСCTTCCACTATCTCCСCСТTTCTCCC CCCATCTGCCTCCAGCCTTGAAGGCTATCCCTTCCAAG +1
\end{abstract}

Fig. 2 Promoter analysis of FocSgel gene showing single GATA transcription factor recognition site

\section{Effects of FocSge1 on hyphal growth, conidiation, hydrophobicity and pigmentation}

The deletion of FocSge1 didn't affect the radial growth of any of the transformants as compared to the wild-type strain. However, it dramatically affected conidial count, colony hydrophobicity and pigmentation. The K09 deletion mutant strain showed reduced aerial mycelium on potato dextrose agar (PDA) medium as compared to the wild-type strain and the full-length complemented strain C7. The truncated mutant T10 showed growth phenotype similar to that of the deletion mutant. Further, the growth characteristics of the wild-type and C7 strains were comparable on PDA, Czapek-Dox and YPD agar media, whereas the K09 and T10 behaved alike (Fig. 4). Since we assume that FocSge1 gene is regulated by nitrogen sources, the effect of different nitrogen sources on Foc growth was then assessed. Different nitrogen sources such as sodium nitrate, ammonium chloride, ammonium nitrate, urea and amino acids such as glutamine and asparagine were used for growth analysis. The kind of growth and pigmentation obtained in wild-type strain was very different from that of the deletion mutant K09 and truncated mutant T10 in all the media. As expected, the phenotype was reverted in the genetic complementation mutant C7. (Fig. 5).

The number of conidia produced by the deletion mutant K09 $\left(2.5 \times 10^{6}\right)$ was significantly reduced when compared with the wild-type strain $\left(3.3 \times 10^{7}\right)$. However, this reduced conidial count was completely restored upon genetic complementation with the full-length FocSge1 gene as evident in the $\mathrm{C} 7$ mutant. The truncated mutant T10 also exhibited low conidial count comparable with the deletion mutant (Fig. 6). Nevertheless, there was no phenotypic abnormality seen in the appearance of the conidia derived from all the three mutants. Furthermore, the conidia produced by all the three mutants were viable and capable of germinating with equal efficiency.

To assess the colony hydrophobicity, a drop of waterbased dye was pipetted onto the mycelium of full-grown plates of the wild-type strain and the three mutants (K09, $\mathrm{C} 7$, and T10). These were observed for percolation of the dye after $5 \mathrm{~min}$. The water-based dye did not percolate into the colonies of wild-type and complementation mutant (C7); however, it completely permeated into the deletion mutant $\mathrm{K} 09$ and truncated mutant T10 indicating that these two mutants have lost their hydrophobicity which is yet another characteristic of the aerial mycelia (Fig. 7a).

To further investigate the role of FocSge1 in pigmentation, the pigments were extracted in two organic solvents; namely, ethyl acetate and chloroform. The amount of pigments obtained in both the solvents derived from the truncated mutant was least, followed by the deletion mutant K09. As visualized by the growth on PDA the amount of pigment in the wild-type strain was highest and was reduced with the deletion of FocSge1 gene; but was completely restored in the complementation mutant C7 (Fig. 7b, c). These results clearly suggest that FocSge1 is involved in mycelial pigmentation. Taken together these results reveal a crucial role for FocSge1 in mycelial development, conidiation, colony hydrophobicity and pigmentation.

\section{FocSge 1 is essential for virulence of $F$. oxysporum f. sp. cubense}

To further investigate the importance of FocSge1 gene in pathogenicity, the Foc race 1 susceptible banana plants 


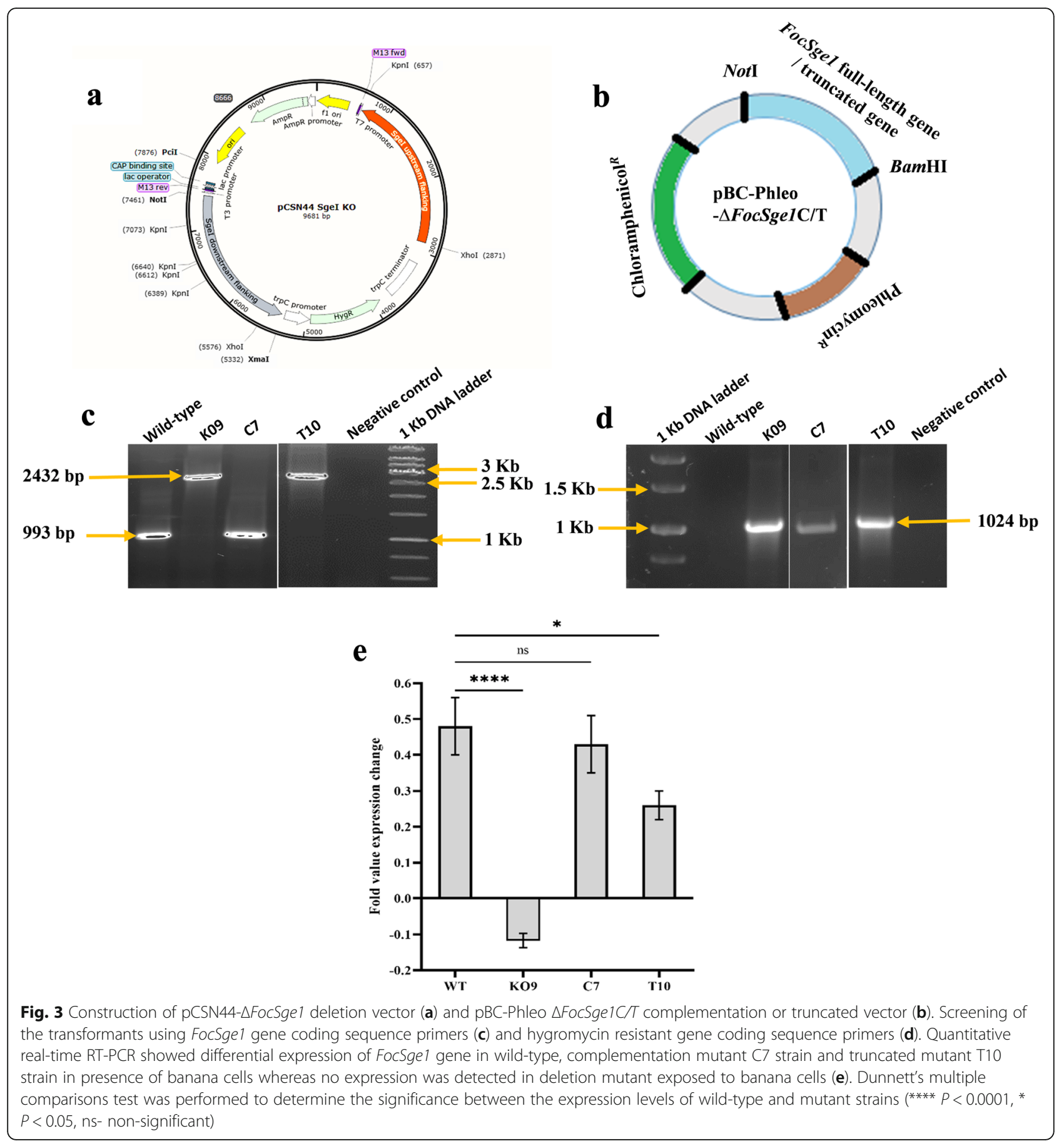

cv. Rasthali were inoculated with four different Foc mass cultures prepared for each mutant and wild-type. This infection-based bioassay was performed in triplicates with a minimum of three plants per experiment per strain. Fusarium wilt symptoms such as yellowing of the older leaves and cracking of pseudostem was evident from third week onwards in the wild-type and C7 mutant inoculated banana plants. However, no such symptoms were observed in plants inoculated with the deletion mutant K09 and truncated mutant T10. After 6 weeks, the plants were photographed and the experiment terminated (Fig. 8a). The banana plants inoculated with wild-type strain showed severe wilt symptoms and completely wilted within the stipulated period; whereas, the plants inoculated with the deletion mutant K09 did not show any external symptoms. However, the lost virulence was restored on genetic complementation as symptoms were comparable in the banana plants infected 


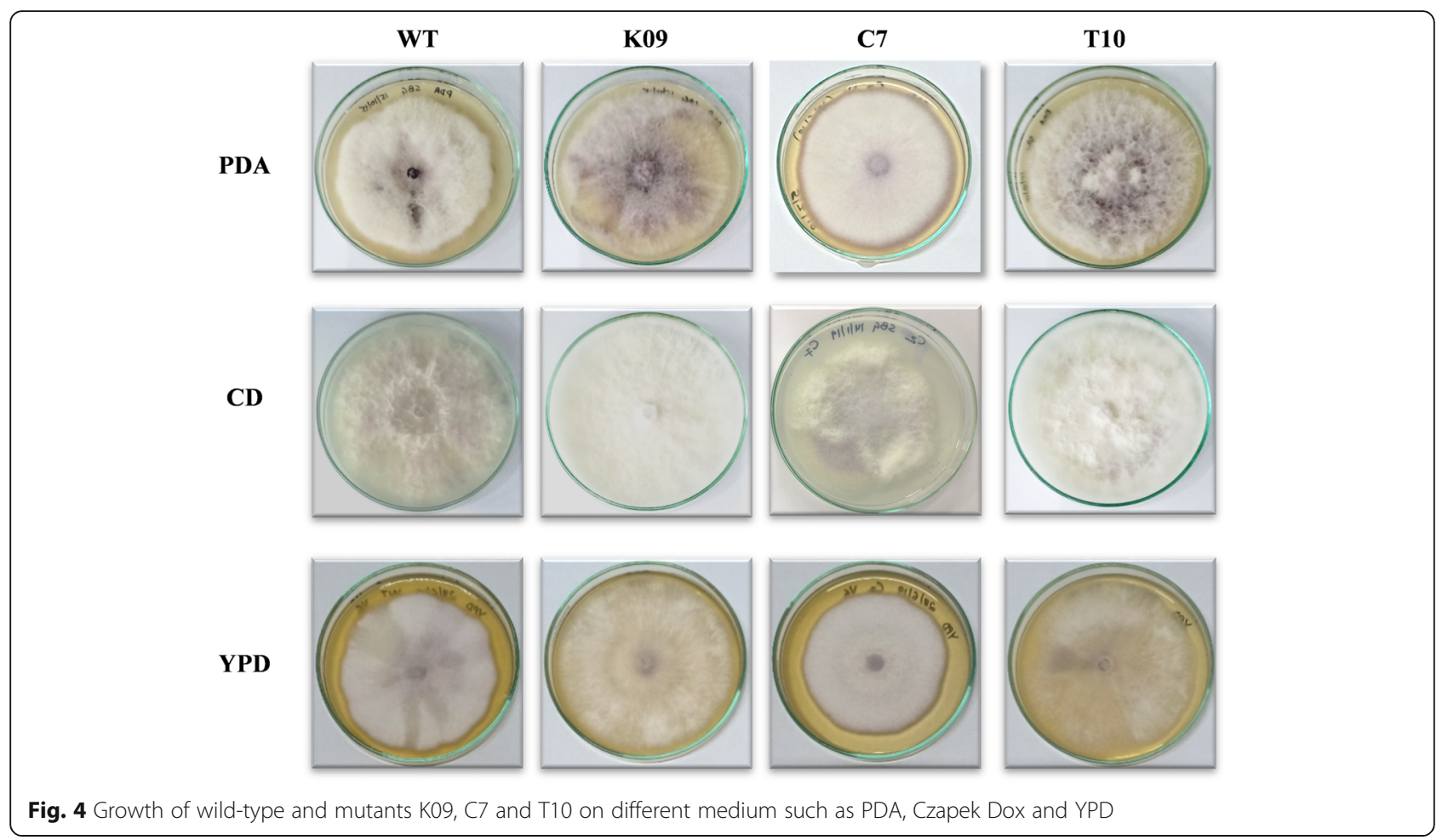

individually with the wild-type and C7 mutant strain. The banana plants inoculated with the truncated mutant T10 also did not show any significant symptoms typical of those seen in the wild-type plant after 6 weeks. The corm tissue of all the inoculated banana plants were cut longitudinally to examine the internal symptoms. Banana plants inoculated with wild-type and C7 mutant showed maximum infestation whereas no visible internal symptoms were observed in the deletion mutant $\mathrm{K} 09$ and truncated mutant T10 (Fig. 8b). Taken together, FocSge1 is required for pathogenicity on banana plants and the C-terminal domain of FocSge1 is essential for this function.

\section{Sensitivity of the FocSge 1 deletion mutant to osmotic stress, oxidative stress and cell wall-damaging agents}

As deletion of FocSge1 gene was known to reduce virulence, its role towards osmotic, oxidative and cell wall stress causing agents was subsequently assessed. There was no statistically significant difference in the radial growth between the wild-type and mutants (at $P$ value $\leq 0.05$ ) in the medium containing osmotic stress agents. However, at $P$ value $\leq 0.1$, there was significant difference between the wild-type and K09 mutant. In the sorbitol-based medium, pigmentation at the colony center was clearly seen in wild-type and C7 mutant, but, was completely absent in other 2 mutants. Moreover, a compact growth was evident in wild-type and C7; whereas, more of dispersed mycelial growth was seen in K09 and T10. (Fig. 9a).
There was statistically significant difference between wild-type and $\mathrm{K} 09$ mutant in the medium containing sodium nitrite. The radial growth of $\mathrm{K} 09$ in minimal medium supplemented with sodium nitrite and menadione was more than the wild-type. However, the sensitivity reverted back to the wild-type in the complementation mutant $C 7$. However, at $P$ value $\leq 0.1$ there was significant difference in the zone of inhibition in medium containing hydrogen peroxide indicating that the deletion mutant is more tolerant to oxidative stress. Additionally, the wildtype and complementation mutant C7 developed pigmentation during incubation. No pigmentation was seen in deletion mutant K09, but slight pigmentation was evident in the truncated mutant T10 with minimum zone of inhibition (Fig. 9b).

To check for the sensitivity of the mutants to cell walldamaging agents, the deletion mutant $\mathrm{K} 09$ and truncated mutant T10 showed resistance and more radial growth in medium supplemented with calcofluor white as compared to the wild-type strain. The genetic complementation mutant $\mathrm{C} 7$ showed a phenotype similar to the wild-type (Fig. 9c).

\section{Fusaric acid analysis}

Fusaric acid produced by the wild-type and mutant strains in Czapek Dox broth was extracted using ethyl acetate and half the fraction evaporated and dissolved in methanol. This methanol fraction when tested for Bacillus subtilis sensitivity, no zone of inhibition was 


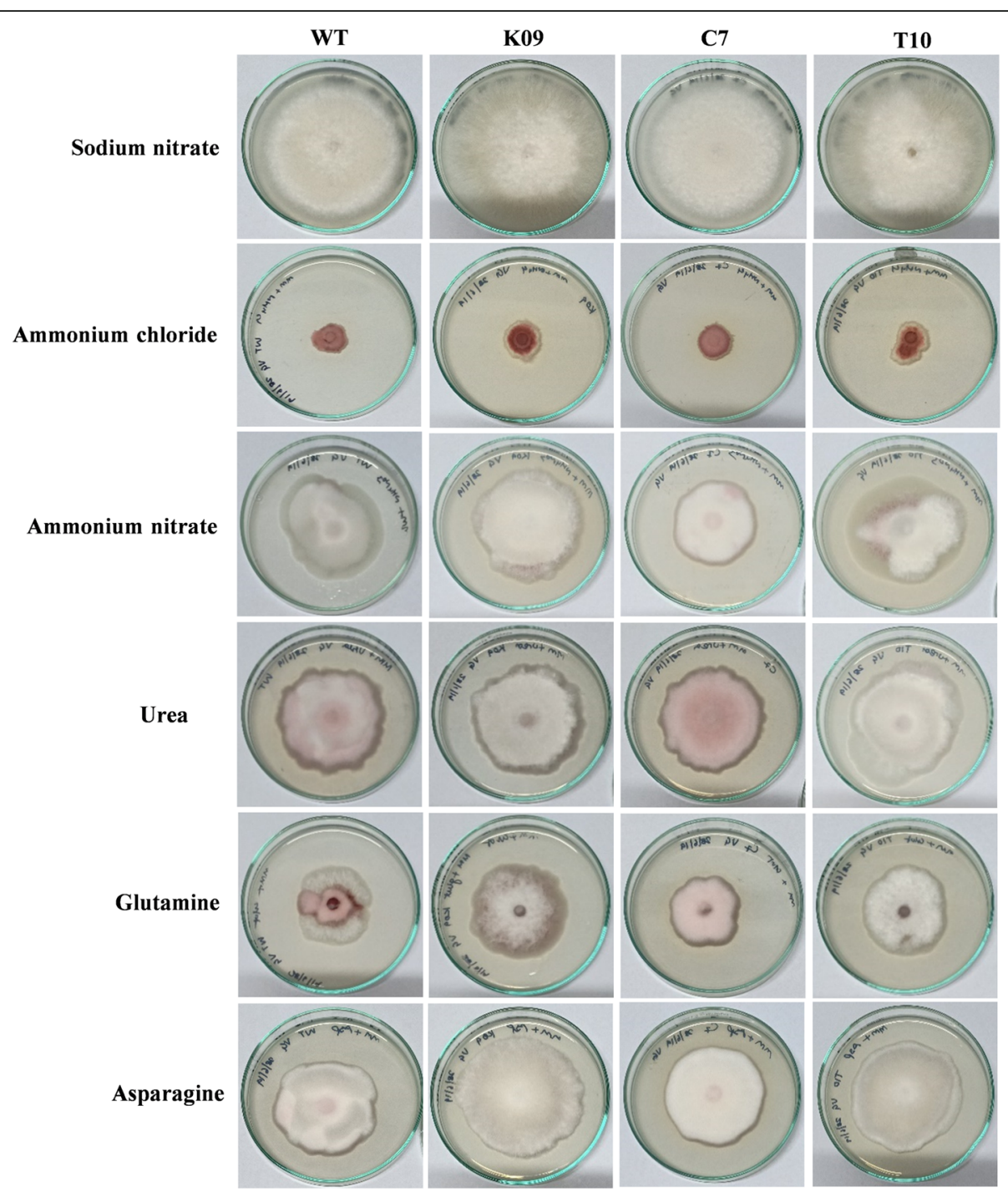

Fig. 5 Growth morphology of the wild-type strain and mutants K09, C7 and T10 on minimal medium supplemented with different nitrogen sources

observed. When the remaining half was extracted using acidified water and diethyl ether and tested for Bacillus subtilis sensitivity, a clear zone of inhibition was observed. The diameter of the zone of inhibition was highest for fusaric acid obtained from wild-type strain, followed by complementation C7 and truncated T10 mutants. A smaller zone of inhibition was observed when fusaric acid produced from deletion strain was used, indicating less production of fusaric acid (Fig. 10). These results thus indicate that FocSgel gene regulates the biosynthesis of fusaric acid to some extent.

\section{Discussion}

Fusarium wilt disease of banana is a notoriety in the agricultural history known to mankind and is still threatening banana production worldwide. The rampant spread of Foc both race 1 and Tropical race 4 all throughout the major banana growing regions is creating a havoc [1]. Almost all the varieties grown for domestic consumption and export are susceptible to these pathogenic strains. Moreover, the physical and chemical methods of management are insufficient. The infected banana fields are rendered useless for fresh plantations as the dormant chlamydospores survive in the soil for decades. Besides, Foc also survives in the asymptomatic alternate hosts and does not show any Fusarium wilt symptoms [11]. Foc shifts its lifestyle from saprophytic to parasitic mode after coming in contact with the host roots which is associated with major alteration in expression and metabolite profile. This change is brought about by a few master regulators that perceive the signals and control the expression of downstream repertoire of genes required for pathogenicity [5]. FocSge1 is one such master regulator that triggers the expression of 


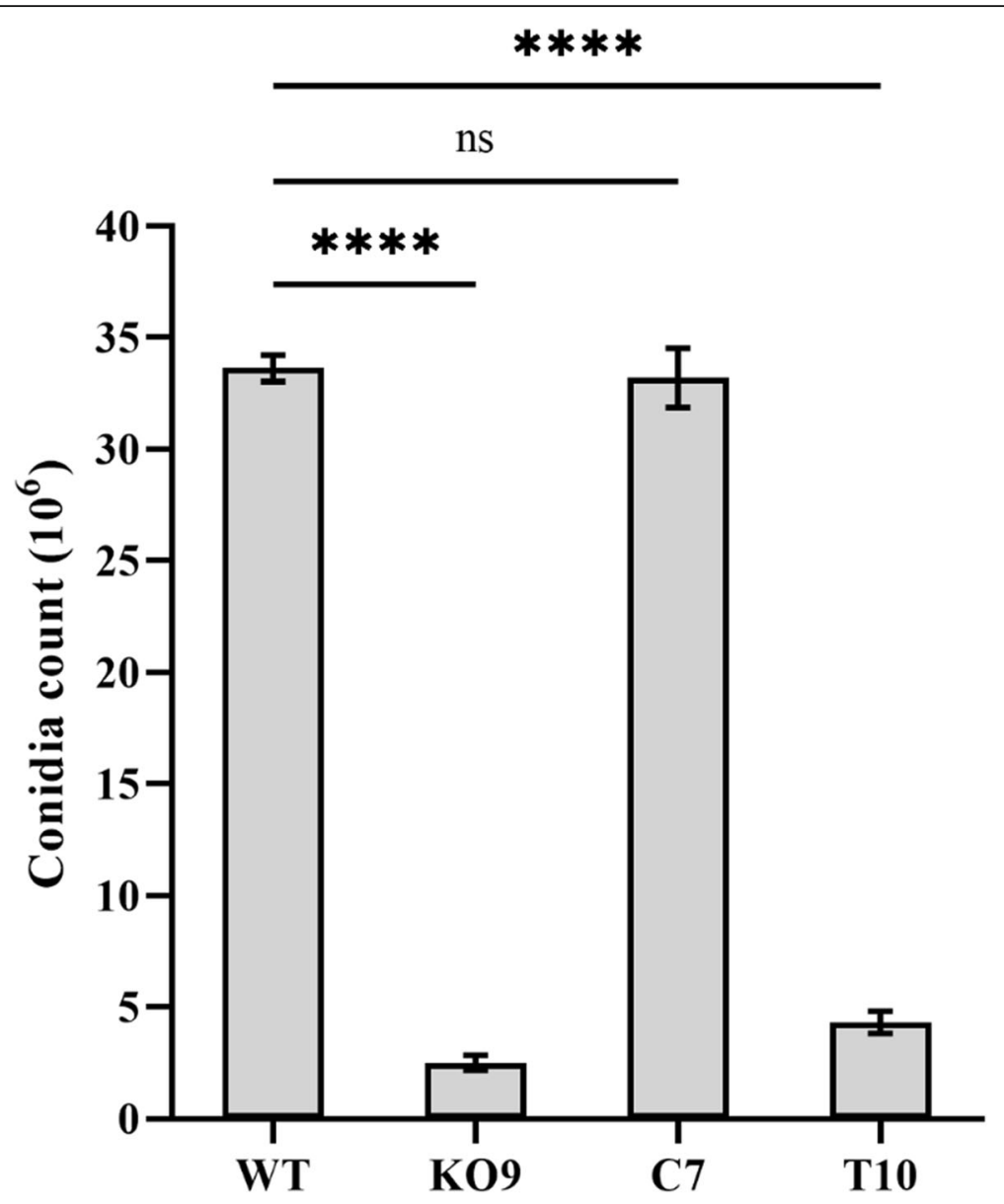

Fig. 6 Conidial count of the wild-type strain and mutants K09, C7 and T10 where deletion mutant and truncated mutant demonstrated reduced conidial count. Dunnett's multiple comparisons test was performed to determine the significance between the conidial count of wild-type and mutant strains (**** $P<0.0001$, ns- non-significant)

a

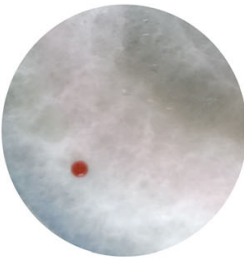

WT

b

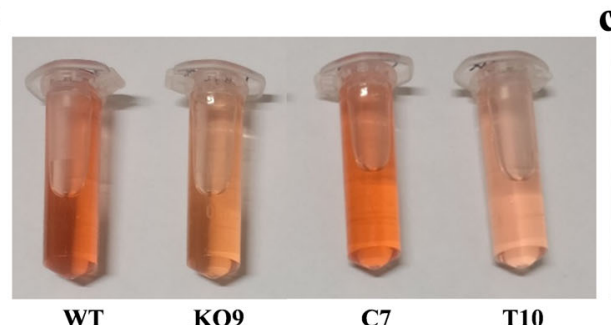

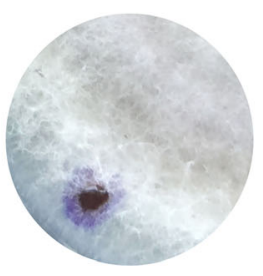

KO9

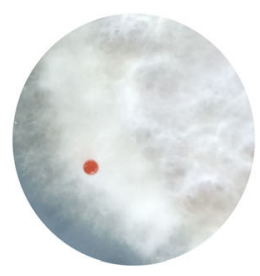

C7

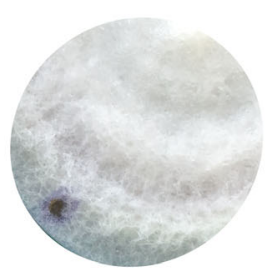

T10

c

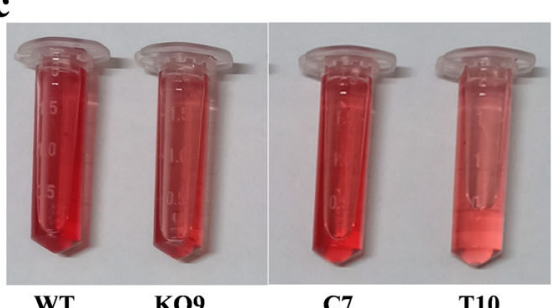

Fig. 7 Qualitative analysis of colony hydrophobicity and pigmentation. The deletion mutant K09 and truncated mutant T10 showed loss of surface hydrophobicity as the dye percolated into the colony within 5 min whereas in wild-type and complementation mutant it was retained as droplet on the surface (a). Reduced pigmentation was observed in K09 and T10 mutants extracted in both ethyl acetate (b) and chloroform (c) 


\section{$\mathbf{a}$}

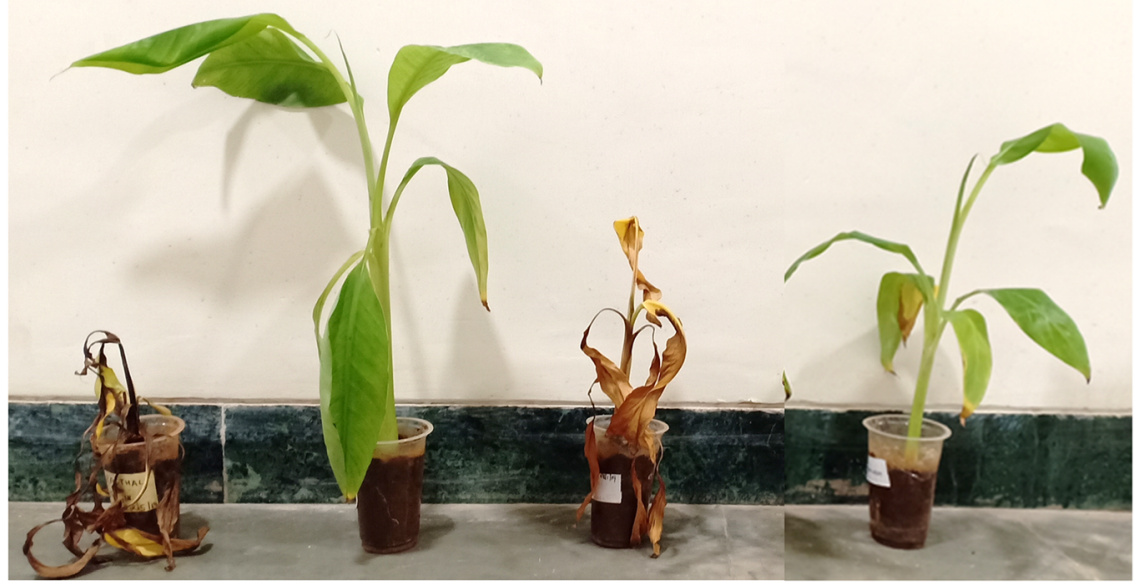

b

WT

KO9

C7

T10

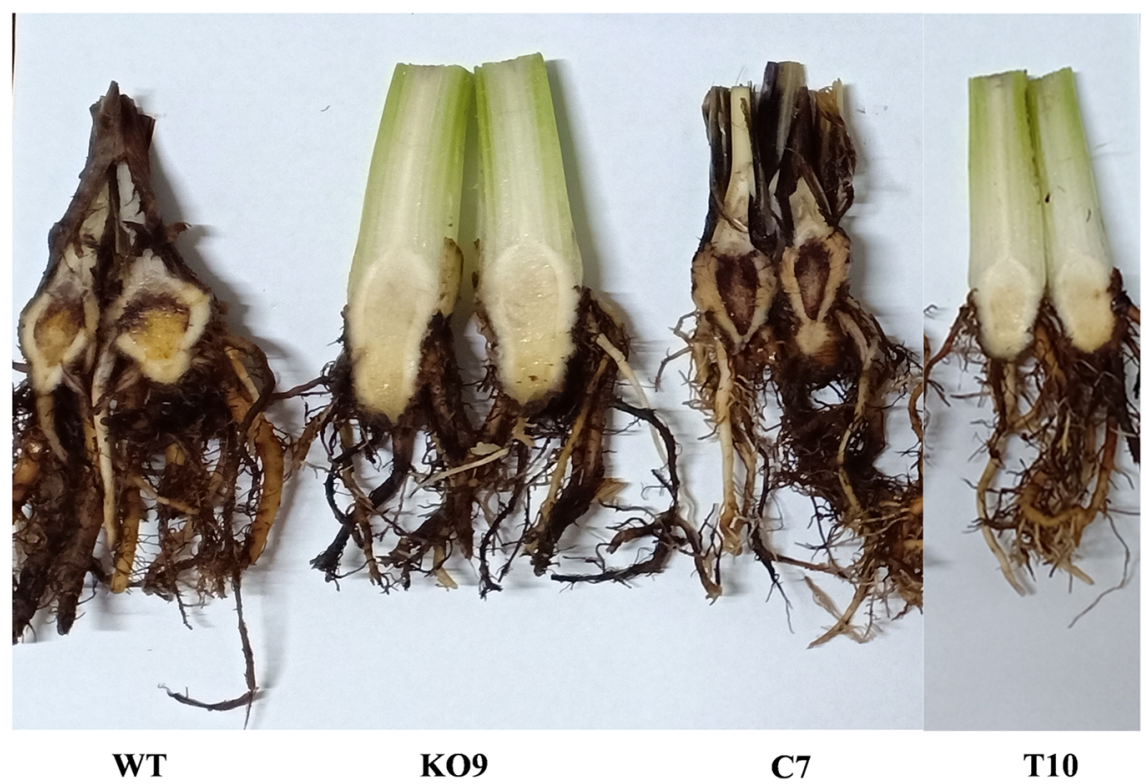

Fig. 8 Ex vivo bioassay using susceptible banana plants. Banana plants inoculated with the wild-type and mutant strains were observed for Fusarium wilt symptoms 6 weeks post inoculation wherein the plants inoculated with wild-type and C7 mutant showed severe wilting. The plants inoculated with deletion mutant K09 and truncated mutant T10 showed no external symptoms (a). Longitudinal sections of the corm tissue displayed internal symptoms such as discoloration and necrosis. Severe infection was seen in plants inoculated with wild-type and C7 complementation mutant whereas plants inoculated with deletion and truncated mutants showed clean corm tissue (b)

effector genes upon colonization. Understanding the molecular basis of Fusarium wilt disease remains the major goal in plant-pathogen interaction research thereby paving way for the development of novel strategies in disease management. In the present study, we examined the roles of FocSge1 gene in Foc race 1 that is known to infect many cultivars of banana. Disruption of FocSge1 gene resulted in several phenotypic variations as compared to the wild-type strain. Moreover, the deletion mutant displayed loss of virulence in susceptible banana variety. We also generated complementation mutants $\mathrm{C7}$ and $\mathrm{T} 10$ in the course of the experiments; where $\mathrm{C} 7$ being a complete rescue. The T10 mutant truncated at its C-terminal domain was created to demonstrate its critical role. To further assess the expression of FocSge1 gene in wild-type and mutant strains, they were inoculated into banana cell suspension cultures. Since the effector genes are regulated even in the presence of plant cells, banana cells were chosen in this assay [5]. Expression of FocSge1 was recorded in wild-type and complementation strains exposed to banana cells indicating that FocSge1 responds to the presence of banana cells. Moreover, expression of the truncated form of FocSge1 was also observed indicating that it is expressed but the 

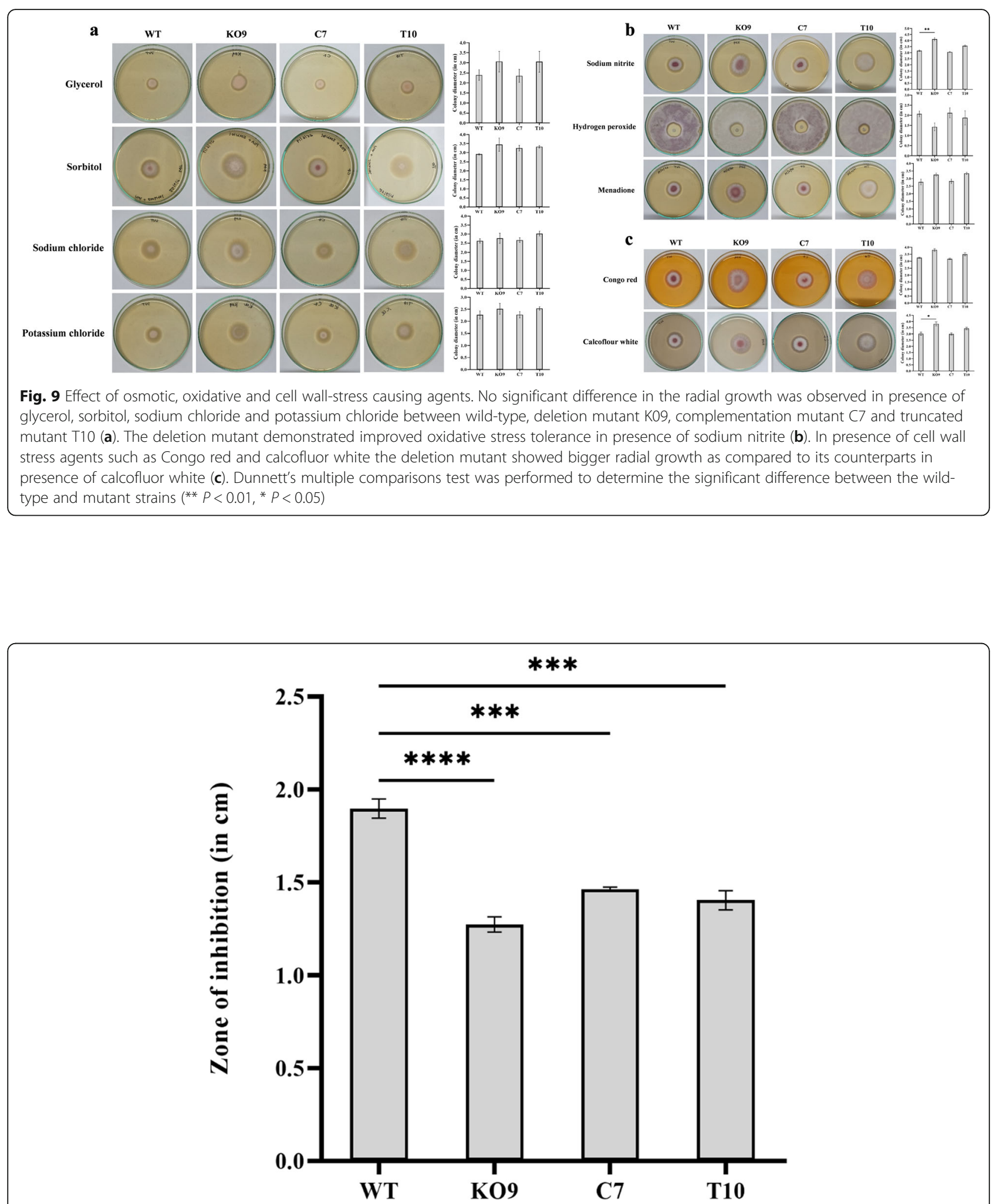

Fig. 10 Qualitative analysis of fusaric acid produced by the strains in Czapek Dox broth. The fusaric acid extracted from the mycelia of wild-type and mutants were tested for their activity against Bacillus subtilis and the zone of inhibition recorded. The zone of inhibition corresponds to the amount of fusaric acid produced. The deletion mutant showed relatively smaller zone of inhibition as compared to the wild-type strain. Dunnett's multiple comparisons test was performed to determine the significant difference between the zone of inhibition of the wild-type and mutant strains $\left({ }^{* * *} P<0.0001,{ }^{* * *} P<0.001\right)$ 
deleted C-terminal domain is crucial for the lost phenotypic characteristics seen in wild-type and the complementation strains. Thus, our study suggests that FocSge1 gene is critical for pathogenicity and regulation of normal growth and development.

Sge1, a homolog of Worl is required for parasitic growth and virulence in Fusarium oxysporum f. sp. lycopercisi (Fol) that infects tomato [5]. Deletion or inactivation of SGE1 resulted in reduced pathogenicity and was correlated with the reduced levels of toxin production and effector proteins. SGE1 from Foc TR4 was shown to regulate series of proteins required for infection. A SGE1 deletion mutant of Foc TR4 demonstrated impaired conidiation and complete loss of virulence on banana plantlets [6]. However, Sge1 deletion mutant of Fol showed no loss of colonization capability, whereas colonization was compromised in Sge1 mutant of Foc. A homolog of Sge1 in Fusarium verticillioides (SGE1) is required for pathogenicity and affected synthesis of secondary metabolites such as fumonisins and fusarins [7]. Nonetheless, SGE1 in F. verticillioides was not essential for conidiation as it was in Foc and Fol. None of these homologs were shown to affect vegetative growth of the fungus. This indicates that the role of Sge1 is varied in different phytopathogens and a need to elucidate its role for each pathosystem is imminent. Multiple sequence alignment of Sge1 protein sequences from different species revealed that the sequence is highly conserved signifying its importance in virulence. Also, Fo47 strain has additional proline-glutamine residues in the C-terminal domain. Fo47 is an endophyte but a nonpathogenic strain capable of inducing defense response in the host plants. Probably an addition of these prolineglutamine residues might have rendered structural deformity of this protein thereby contributing towards lost pathogenicity. This observation concurs with the loss of pathogenicity of the truncated mutant T10 created in this study.

Some virulence-related genes are induced by nitrogen starvation and also by the kind of nitrogen source provided in the medium [12]. The kind of nitrogen available in planta is different from that in soil and thus there is a differential regulation with respect to the nitrogen source available. In order to find whether FocSge1 is regulated by nitrogen source, we firstly identified the presence of a GATA element in the promoter region of this gene. Nevertheless, the expression of the effector gene SIX1, which is downstream to FocSge1 was observed in medium containing sucrose and potassium nitrate, thus we believe that FocSge1 is in turn regulated by nitrate in the medium [13].

Deletion of FocSge1 gene resulted in altered growth and development in Foc indicating that it influences these biological processes in this fungus. The wild-type strain showed more of a cottony growth and pigmentation on PDA and YPD media, whereas the deletion and truncated mutants displayed poor aerial hyphae and reduced pigmentation. Additionally, the radial growth was comparable with the wild-type strain in all media tested. Since we now know that FocSge1 is differentially regulated by the kind of nitrogen source, we tested the growth phenotype of these mutants in minimal medium supplemented with different nitrogen sources. In all the media tested, the deletion mutant $\mathrm{K} 09$ and truncated mutant T10 displayed altered phenotype as compared to the wild-type strain in terms of mycelial growth, aerial hyphae and pigmentation. This indicates that FocSge1 gene is involved in asexual growth development. Furthermore, the number of conidia in the deletion mutant were considerably less than the wild-type strain. The conidia count was restored when the deletion mutant was complemented with the full-length FocSge1 gene but not in the truncated mutant. This clearly indicates that FocSge1 is required for maintaining the conidial count. In previous study, a similar phenotype was seen in Fusarium oxysporum f. sp. lycopersici where the SGE1 deletion mutants produced about 6-fold less microconidia compared to the wild-type [5] and the Fusarium oxysporum f. sp. cubense TR4 produced about 4-fold less conidia compared with WT [6]. However, SGE1 deletion did not affect conidiation in Fusarium verticillioides, a maize pathogen [7].

The aerial hyphae and the conidia are the ones which provide hydrophobicity to the colony, since there was poor hyphal growth and conidiation, we tested the mutants for hydrophobicity and found that the deletion and truncated mutants completely lost the hydrophobicity. The pigments produced in the wild-type strain were abundant as compared to the deletion and truncated mutants. Also, this lost phenotype was regained in the complementation mutant strain.

To investigate the role of FocSge1 in pathogenesis, 2 month-old hardened susceptible banana plants cv. Rasthali were independently inoculated with the four mass cultures (WT, K09, C7 and T10). The banana plants inoculated with the wild-type strain showed typical Fusarium wilt symptoms whereas the deletion mutant K09 could not infect the banana plants and therefore the plants did not show any external or internal symptoms. However, the virulence was restored by genetic complementation indicating that FocSge1 is required for full virulence. Moreover, the truncated mutant T10 also showed reduced virulence. The series of altered phenotype seen in the deletion mutant including hyphal growth, hydrophobicity, poor conidial count and cell wall integrity may have together contributed to the reduced virulence in the deletion and truncated mutant on the banana plants. We tested the expression of the target gene FocSix1 in the inoculated plants using quantitative RT-PCR where there was expression 
observed in the wild-type and C7 mutant whereas no expression was seen in the K09 and T10 mutant (data not shown).

Rigidity of the fungal cell wall, osmotic and oxidative stress tolerance are important characters required for maintaining cellular integrity, survival and penetration in the host cells [14-16]. Moreover, the phytopathogenic fungi also need to encounter oxidative stress generated while infecting the plant cells [17, 18]. The FocSge1 deletion mutant $\mathrm{K} 09$ generated in this study showed increased tolerance to oxidative stress (sodium nitrite) as compared to the wild-type strain. Since these features are somewhat associated with the cell wall integrity, the tolerance of the mutant to cell wall-damaging agents such as Congo red and calcofluor white were tested. The radial growth in the calcofluor white medium was relatively more in the deletion and truncated mutants as compared to the wild-type strain indicating that they are capable of resisting it. Inappropriate accumulation of trehalose, glycerol and other osmolytes have been known to regulate stress responses [19-22]. Intercellular trehalose plays a critical role in free radical scavenging, whereas glycerol affirmatively responds to osmotic stress [23]. Increased tolerance of the deletion and truncated mutants to oxidative and cell-wall stress may be due to altered cell wall features and composition and thus shows that FocSge1 is required for maintaining normal cell wall integrity. Moreover, HOG pathway is involved in stress management and accumulation of compatible solutes in the cell [24]. Based on the results obtained in our study we assume that FocSge1 gene regulate the HOG pathway at some point and thus deletion of FocSge1 resulted in loss of control on the HOG pathway imparting more tolerance to stress agents in deletion mutant as compared to wild-type and complementation mutant. Further the role of FocSge1 in fusaric acid production was assessed as fusaric acid is one of the virulence factor [25]. The fusaric acid production was tested in wild-type and mutant strains, where the deletion mutant showed less production capability as compared to the wild-type and complementation mutants.

\section{Conclusions}

In conclusion, the present work demonstrates that FocSge1 is vital for pathogenesis in banana and is involved in hyphal growth, pigmentation, hydrophobicity, conidiation and fusaric acid production. We demonstrate that the C-terminal domain of FocSge1 is important for its in vivo functioning as the truncated mutant behaved similar to the deletion mutant.

\section{Methods}

\section{Strain and culture conditions}

Fusarium oxysporum f. sp. cubense (Foc) race 1 wildtype isolate (VCG 0125) obtained from the Indian
Institute of Horticulture Research (IIHR), Bangalore (India) was used in all the experiments. It was stored as a conidial suspension in $15 \%$ glycerol at $-80{ }^{\circ} \mathrm{C}$. The wild-type strain and transformants were grown and subcultured on potato dextrose agar (PDA). For experiments such as conidial count, genomic DNA isolation, RNA isolation, fusaric acid extraction, Foc was grown in potato dextrose broth (PDB) at $30^{\circ} \mathrm{C}$ and agitated at 160-200 rpm for 5-10 days.

\section{In silico analysis of FocSge1 gene}

The FocSge1 (FOC1_g10010072) sequence was identified in the whole genome shotgun sequencing database (KB730034 AMGP01000000) of Fusarium oxysporum f. sp. cubense race1 (scaffold56, whole genome shotgun sequence) available with the NCBI database. The FocSge1 amino acid sequence was aligned with its orthologs using the online Clustal ${ }^{\text {mi }}$ Omega tool. Two kilobases upstream sequence (the promoter region) of FocSge1 gene was screened for the presence of GATA sequence which is known to be responsive to different nitrogen sources in the medium.

\section{Generation of FocSge1 deletion mutants}

The FocSge1 deletion vector was generated by inserting the upstream and downstream flanking sequences of FocSge1 onto the two sides of the hygromycin resistance gene cassette. The FocSge1 upstream sequence (2047 bp) along with $162 \mathrm{bp}$ of the coding region was amplified using FocSge1 UPS Fw/FocSge1 UPS Rv primer set and inserted into the KpnI and XhoI restriction sites of the pCSN44 vector backbone upstream to the hygromycin cassette. Subsequently, the downstream flanking sequence of $1998 \mathrm{bp}$ of FocSge1 along with $124 \mathrm{bp}$ of coding sequence was amplified using FocSge1 DWS Fw/ FocSge1 DWS Rv primer pair and cloned downstream of the hygromycin cassette between NotI and XmaI sites to form the pCSN44- $\Delta$ FocSge1 deletion vector. Both upstream and downstream DNA fragment of FocSge1 gene were sequenced. This pCSN44- $\Delta$ FocSge1 deletion vector was transformed into Foc spheroplasts.

The spheroplasts were prepared by growing the Foc culture on potato dextrose agar at $30^{\circ} \mathrm{C}$ for $8-12$ days. Conidia were harvested using Tween 80 solution $(0.005 \% \mathrm{v} / \mathrm{v})$ and separated from the mycelia by filtering through the absorbent cotton bed. Approximately, $10^{8}$ conidia $/ \mathrm{mL}$ were inoculated into potato dextrose broth and incubated at $30^{\circ} \mathrm{C}$ and agitated at $200 \mathrm{rpm}$ for 22 $23 \mathrm{~h}$. The following day, mycelia was separated using four layers of cheese-cloth and washed several times with chilled distilled water. Further the mycelia was washed with chilled osmotic medium $\left(0.27 \mathrm{M} \mathrm{CaCl}_{2}\right.$ and $0.6 \mathrm{M} \mathrm{NaCl})$. The washed mycelia was then transferred to a flask containing osmotic medium, lysing enzyme 
[900 mg/mL] (Novozyme, USA) and BSA fraction V [60 $\mathrm{mg} / \mathrm{mL}$ ] (MP Biomedicals, USA) and mixed to form a homogenous slurry. This mixture was then incubated in water bath at $37^{\circ} \mathrm{C}$ for $2-3 \mathrm{~h}$. The reaction mixture was swirled vigorously to release protoplasts from the mycelial debris. This reaction mix was then filtered through Mira cloth (Merck, USA) to separate the spheroplasts from the mycelial debris. The spheroplasts were then pelleted by centrifuging at $1650 \mathrm{~g}$ in a swinging bucket rotor for $10 \mathrm{~min}$. The pellet was washed with $1 \mathrm{X}$ STC solution (1.2 M D-sorbitol, $50 \mathrm{mM} \mathrm{CaCl} 2$ and $35 \mathrm{mM}$ $\mathrm{NaCl}, 10 \mathrm{mM}$ Tris). The supernatant was discarded and pellet was resuspended in $1 \mathrm{X}$ STC. Five microgram of the linearized plasmid DNA (using PciI) along with equal volume of $2 \mathrm{X} \mathrm{STC}, 50 \mu \mathrm{l} 50 \% \mathrm{w} / \mathrm{v}$ PEG ( $\mathrm{t}$ ) (2X STC: $50 \%$ w/v PEG 8000::1:1) and W7 hydrochloride $(5 \mu \mathrm{g} / \mathrm{mL})$ [26] was added to the spheroplasts and incubated on ice for $30 \mathrm{~min}$. After incubation $1 \mathrm{~mL}$ of PEG(t) was added to the mixture and further incubated at room temperature for $30 \mathrm{~min}$. This mixture was then spread onto PDA medium containing sucrose $(1.329 \mathrm{M})$ and incubated at room temperature. After $16-18 \mathrm{~h}$, the plates were overlaid with PDA medium supplemented with hygromycin $(50 \mu \mathrm{g} / \mathrm{mL})$ and incubated at $30{ }^{\circ} \mathrm{C}$ for $2-3$ days till the appearance of transformants on the plates. The putative transformants were then grown on PDA medium supplemented with hygromycin and screened by PCR using primers FocSgelcds Fw and FocSgelcds Rv. A single confirmed transformant was selected and used for further analysis.

\section{Complementation of the FocSge1 gene deletion mutant with full-length gene and truncated version}

To functionally annotate FocSge1 gene, the deletion mutant strain was complemented with the full-length FocSge1 gene. The complementation vector was constructed by PCR amplifying the locus having $2000 \mathrm{bp}$ upstream promoter region, $993 \mathrm{bp}$ coding sequence and 1000 bp downstream terminator region using FocSge1 Comp Fw/FocSge1 Comp Rv primer pair. This $4054 \mathrm{bp}$ amplified sequence was cloned into NotI and BamHI site of $\mathrm{pBC}-\mathrm{Phleo}$ vector to give $\mathrm{pBC}-\mathrm{Phleo}-\Delta$ FocSge1C. The FocSge1 gene in $\mathrm{pBC}$-Phleo vector was sequenced. This vector was further linearized using $B m t \mathrm{I}$ and transformed into Foc spheroplast as described above. The transformants were selected on the medium supplemented with phleomycin and hygromycin. The transformants were sub-cultured for seven generations, further prepared into single spore suspension and stored until further use. The putative transformants were screened for the complementation construct using PCR. Simultaneously, a truncated version (pBCPhleo- $\Delta$ FocSge1T) of FocSge1 gene was created using primer pair FocSge1CompTRN Fw/ FocSge1CompTRN Rv, wherein the 70 amino acids (210 bp) C-terminal domain was deleted. The $\mathrm{pBC}$-Phleo- $\Delta$ FocSge1T vector was linearized using ApaI and transformed into the deletion mutant as described previously to investigate its importance.

\section{Quantitative reverse transcription PCR (qRT-PCR) analysis}

To validate FocSge1 expression in the wild-type and mutant strains of Foc, $10^{5}$ conidia were inoculated into cell suspension cultures of banana cv. Rasthali. Inoculated and un-inoculated control flasks were incubated at $25^{\circ} \mathrm{C}, 80 \mathrm{rpm}$ for $60 \mathrm{~h}$. Cultures were harvested by centrifugation and total RNA was extracted as previously described in [27]. The total RNA was purified using RNeasy mini kit (Qiagen, Netherlands) and DNA was removed using on-column DNase treatment, following the manufacturers' instructions. Total RNA $(1 \mu \mathrm{g})$ was used to prepare cDNA with ProtoScript ${ }^{\circ}$ First Strand cDNA Synthesis Kit (New England Biolabs MA, USA) following manufactures' protocol. Fifty nanograms of cDNA was used as template for expression analysis of FocSge1 gene using Sge1RT Fw and Sge1RT Rv primers. qRT-PCR was performed using CFX96 Real Time System (Bio-Rad, California, United States) and SsoFast EvaGreen ${ }^{\circ}$ Supermix (Bio-Rad, California, United States). The Foc translation elongation factor $1 \alpha$ (TEF-1 $\alpha$ ) was used as an endogenous reference control for normalization and reactions were carried out in triplicates. The relative expression levels were determined using REST-MCS software [28].

\section{Mycelial growth on different medium}

Foc wild-type and mutant culture plugs were obtained from 10 days-old PDA plate and inoculated on different media such as potato dextrose agar (PDA), Czapek Dox agar, yeast extract peptone dextrose agar (YPD) and minimal media containing different nitrogen sources (glutamine, urea, sodium nitrate, ammonium chloride, ammonium nitrate). These plates were incubated at $30^{\circ} \mathrm{C}$ for 8 days. The plates were observed and documented for growth, colony morphology and pigmentation.

\section{Effect on pigmentation and conidiation}

Conidia were harvested from a 10 days-old Foc plate and counted using hemocytometer. Further, $10^{3}$ conidia were inoculated in $50 \mathrm{~mL}$ of $\mathrm{PDB}$. This was incubated at $30^{\circ} \mathrm{C}, 160 \mathrm{rpm}$ for 10 days. Mycelia was separated using four layers of cheese cloth and ground into fine powder using liquid nitrogen. This powdered mycelia was then equally divided into two $15 \mathrm{~mL}$ tubes containing $10 \mathrm{~mL}$ each of ethyl acetate and chloroform. The tubes were incubated on a rotary shaker overnight at room temperature. The crushed mycelia were separated by centrifuging at $8228 \mathrm{~g}$ for $20 \mathrm{~min}$ at room temperature. This experiment was repeated at least thrice each with three technical replicates. 


\section{Effect on colony hydrophobicity}

Foc wild-type and mutant plugs were obtained from 10 days-old PDA plate and inoculated on PDA medium. The plates were incubated at $30^{\circ} \mathrm{C}$ for $8-10$ days and used for hydrophobicity testing. Ten microliters of a water-based dye was pipetted onto the colonies and allowed to rest at room temperature for $5 \mathrm{~min}$. The percolation of the dye was observed and plates were photographed.

\section{Pathogenicity assay}

The ex vivo bioassay was carried out using 2 months-old hardened tissue culture banana plants cv. Rasthali (AAB 'Silk' group) (derived from the micropropagation of banana from embryogenic callus) and Foc mass culture $[29,30]$. Rasthali plants were obtained from fields and authenticated by the Karnataka Horticulture Department, Govt. of India. For Foc mass culture, wild-type Foc strain and the other two mutants were grown in PDB for 10 days at $30^{\circ} \mathrm{C}$ at $160 \mathrm{rpm}$. Five hundred milliliters of Foc culture was inoculated in the mixture of autoclaved sand and maize bran in the ratio of 19:1. This mixture was incubated at room temperature for 4 weeks and used for infection. The hardened banana plants were uprooted, roots injured using needle and transplanted in the mixture of soil and Foc mass culture in 1:1 ratio. These plants were incubated under controlled conditions and observed for symptoms after 6 weeks. Three plants were inoculated with each strain and the experiment was repeated thrice. After 6 weeks, the plants were uprooted and corm tissue was sectioned longitudinally to observe internal symptoms.

\section{Sensitivity of the FocSge1 deletion mutant to osmotic stress, oxidative stress and cell wall-damaging agents}

To test the sensitivity of the Foc wild-type strain and mutants against osmotic, oxidative and cell wall stress agents, the conidia were allowed to grow in medium containing the stressors. For osmotic stress experiments, glycerol $(0.8 \mathrm{M})$, sorbitol $(1.0 \mathrm{M}), \mathrm{KCl}(1 \mathrm{M})$ and $\mathrm{NaCl}$ $(0.8 \mathrm{M})$ were added individually to the medium. For inducing oxidative stress, menadione $(10 \mu \mathrm{g} / \mathrm{mL})$ and sodium nitrite $(1 \mathrm{mM})$ were added individually to the medium. For hydrogen peroxide assay, $10^{3}$ conidia were spread on the plate and incubated at $30^{\circ} \mathrm{C}$ overnight. Following day, sterile paper discs loaded with $3 \%$ hydrogen peroxide was placed in the center of the plate and incubated further at $30^{\circ} \mathrm{C}$ for 2 days. For cell wall stress, Congo red $(50 \mu \mathrm{g} / \mathrm{mL})$ and calcoflour white $(40 \mu \mathrm{g} / \mathrm{mL})$ was used and mixed directly into the medium [31,32]. Conidia were harvested from 10 days-old Foc wild-type and mutant plates and $10^{3}$ conidia were inoculated in the center of each plate. Plates were incubated at $30^{\circ} \mathrm{C}$ for 3 days. For all the assays, radial growth was measured; while, for hydrogen peroxide assay, the zone of inhibition was measured and the difference was represented in graphical format.

\section{Detection of Fusaric acid production}

Conidia were harvested from a 10 days-old PDA plate and $10^{3}$ spores were inoculated in $500 \mathrm{~mL}$ Czapek Dox broth and further incubated at $30^{\circ} \mathrm{C}$ at $160 \mathrm{rpm}$ for 10 days. Mycelia was separated using cheese cloth and $1 \mathrm{~g}$ (wet weight) was crushed to a fine powder using liquid nitrogen. Fusaric acid was extracted in ethyl acetate solvent using Soxhlet [33]. The ethyl acetate fraction containing fusaric acid was then divided into two parts; one part was evaporated to dryness and reconstituted in 2 $\mathrm{mL}$ of methanol; whereas, the other was evaporated to dryness and reconstituted in $25 \mathrm{~mL}$ distilled water $(\mathrm{pH}$ 3.0 adjusted using $3 \mathrm{~N} \mathrm{HCl}$ ). Further, it was extracted with an equal volume of ethyl ether 3 times that was evaporated to dryness and the residue was finally reconstituted in $2 \mathrm{~mL}$ methanol. Overnight grown culture of Bacillus subtilis was used to test the presence of fusaric acid. The Bacillus subtilis culture $\left(0.1 \mathrm{OD}_{600 \mathrm{~nm}}\right)$ was swabbed on the nutrient agar plate, wells bored and $50 \mu \mathrm{L}$ of the extract was added to each well. These plates were incubated overnight at $30^{\circ} \mathrm{C}$ and observed for the zone of inhibition.

\section{Statistical analysis}

To determine the statistical significance between the two groups for all experimental values Dunnett's multiple comparisons test was carried out using GraphPad Prism 8.0.2. ( $P$ value $\leq 0.05)$.

\section{Supplementary information}

Supplementary information accompanies this paper at https://doi.org/10. 1186/s12866-020-01936-y.

\section{Additional file 1: Table S1. Primers used the study.}

Additional file 2: Figure S1. Multiple sequence alignment of protein sequences of FocSge1 with gluconate transporter inducer Gti1 from S. pombe (NP_592911), Bcreg1 from Botrytis cinerea (XP_024547925), Ryp1 from Histoplasma capsulatum (ABX74945.1) and Wor1p from Candida albicans SC5314 (AOW26645.1).

Additional file 3: Figure S2. Multiple sequence alignment of protein sequences of FocSge1 with its homologs from Fusarium oxysporum f. sp. lycopersici 4287 (XP_018248224), Fusarium sp. FOSC 3-a (EWY91661), Fusarium oxysporum f. sp. pisi HDV247 (EXA45922), Fusarium oxysporum Fo5176 (EGU84598) and Fusarium oxysporum Fo47 (EWZ38328).

\section{Additional file 4}

\section{Abbreviations}

Foc: Fusarium oxysporum f. sp. cubense; f. sp.: Formae speciales; TR4: Tropical race 4; Fol: Fusarium oxysporum f. sp. Iycopersici; SGE1: SIX Gene Expression 1; SIX1: Secreted In xylem 1; aa: Amino acids; Kbp: Kilo base pairs;

PCR: Polymerase Chain Reaction; PDA: Potato Dextrose Agar; PDB: Potato Dextrose Broth; YPD: Yeast Extract Peptone Dextrose Agar; cv: Cultivar; VCG: Vegetative Compatible Groups; qRT-PCR: Quantitative Real-Time PCR 


\section{Acknowledgements}

SBG thanks Prof. Jacinta S. D'Souza (Chairperson, School of Biological Sciences, UM-DAE Centre for Excellence in Basic Sciences) and Prof. Narayan S. Punekar (Department of Bioscience and Bioengineering, Indian Institute of Technology-Bombay, Mumbai) for their support, valuable inputs and critical reading of the manuscript.

\section{Authors' contributions}

VG and SBG conceived the study and conducted the experimental investigation.VG and SBG interpreted data and wrote the original draft of the manuscript. TRG provided some materials for this study, reviewed the manuscript and provided valuable suggestions. All authors commented on the manuscript and approved the final manuscript.

\section{Funding}

The corresponding author SBG received the research grant from the Department of Science and Technology, Ministry of Science and technology, India under the DST-INSPIRE Faculty scheme (DST/INSPIRE FACULTY AWARD/ IFA15-LSPA32). The funding organization was not involved in the design of the study, analysis, or interpretation of data, or in writing of the manuscript.

\section{Availability of data and materials}

All data analyzed during this study are included in this article. The FocSgel coding sequence is available in the National Center for Biotechnology Information (NCBI) database with accession number MT797862.

\section{Ethics approval and consent to participate}

Not applicable.

\section{Consent for publication}

Not applicable.

\section{Competing interests}

The authors declare that they have no competing interests.

\section{Author details}

${ }^{1}$ School of Biological Sciences, UM-DAE Centre for Excellence in Basic Sciences, University of Mumbai campus, Kalina, Santacruz (E), Mumbai 400 098, India. ${ }^{2}$ Plant Cell Culture Technology Section, Nuclear Agriculture \& Biotechnology Division, Bhabha Atomic Research Centre, Trombay, Mumbai 400 085, India.

\section{Received: 13 January 2020 Accepted: 4 August 2020}

\section{Published online: 14 August 2020}

\section{References}

1. Ghag SB, Shekhawat UK, Ganapathi TR. Fusarium wilt of banana: biology, epidemiology and management. Int J Pest Manag. 2015;61:250-63.

2. Dita M, Barquero M, Heck D, Mizubuti ES, Staver CP. Fusarium wilt of banana: current knowledge on epidemiology and research needs toward sustainable disease management. Front Plant Sci. 2018;9:1468.

3. Jonkers W, Dong Y, Broz K, Kistler HC. The Wor1-like protein Fgp1 regulates pathogenicity, toxin synthesis and reproduction in the phytopathogenic fungus Fusarium graminearum. PLoS Pathog. 2012;8:e1002724.

4. Michielse CB, Becker M, Heller J, Moraga J, Collado IG, Tudzynski P. The Botrytis cinerea Reg1 protein, a putative transcriptional regulator, is required for pathogenicity, conidiogenesis, and the production of secondary metabolites. Mol Plant-Microbe Interact. 2011;24:1074-85.

5. Michielse CB, van Wijk R, Reijnen L, Manders EM, Boas S, Olivain C, et al. The nuclear protein Sge1 of Fusarium oxysporum is required for parasitic growth. PLoS Pathog. 2009;5:e1000637.

6. Hou X, An B, Wang Q, Guo Y, Luo H, He C. SGE1 is involved in conidiation and pathogenicity of Fusarium oxysporum f. sp. cubense. Can J Microbiol. 2018;64:349-57.

7. Brown DW, Busman M, Proctor RH. Fusarium verticillioides SGE1 is required for full virulence and regulates expression of protein effector and secondary metabolite biosynthetic genes. Mol Plant-Microbe Interact. 2014a;27:809-23.

8. Widinugraheni S, Niño-Sánchez J, van der Does HC, van Dam P, GarcíaBastidas FA, Subandiyah S, et al. A SIX1 homolog in Fusarium oxysporum $\mathrm{f}$. sp. cubense tropical race 4 contributes to virulence towards Cavendish banana. PLoS One. 2018;13:e0205896.
9. Li E, Wang G, Xiao J, Ling J, Yang Y, Xie B. A SIXI homolog in Fusarium oxysporum f. sp. conglutinans is required for full virulence on cabbage. PLoS One. 2016;11:e0152273.

10. Rep M, Van Der Does HC, Meijer M, Van Wijk R, Houterman PM, Dekker HL, et al. A small, cysteine-rich protein secreted by Fusarium oxysporum during colonization of xylem vessels is required for 1-3-mediated resistance in tomato. Mol Microbiol. 2004;53:1373-83.

11. Ploetz RC. Fusarium wilt of banana. Phytopathology. 2015;105:1512-21.

12. López-Berges MS, Rispail N, Prados-Rosales RC, Di Pietro A. A nitrogen response pathway regulates virulence functions in Fusarium oxysporum via the protein kinase TOR and the bZIP protein MeaB. Plant Cell. 2010;22:2459-75.

13. van der Does HC, Duyvesteijn RG, Goltstein PM, van Schie CC, Manders EM, Cornelissen BJ, et al. Expression of effector gene $S I X 1$ of Fusarium oxysporum requires living plant cells. Fungal Genet Biol. 2008;45:1257-64.

14. Chen Z, Nunes MA, Silva MC, Rodrigues CJ Jr. Appressorium turgor pressure of Colletotrichum kahawae might have a role in coffee cuticle penetration. Mycologia. 2004;96:1199-208.

15. Arana DM, Prieto D, Román E, Nombela C, Alonso-Monge R, Pla J. The role of the cell wall in fungal pathogenesis. Microb Biotechnol. 2009;2:308-20.

16. Gow NA, Latge JP, Munro CA. The fungal cell wall: structure, biosynthesis, and function. Microbiol Spectr. 2017;5:1 FUNK-0035-2016.

17. Heller J, Tudzynski P. Reactive oxygen species in phytopathogenic fungi: signaling, development, and disease. Annu Rev Phytopathol. 2011;49:369-90.

18. Shalaby S, Horwitz BA. Plant phenolic compounds and oxidative stress: integrated signals in fungal-plant interactions. Curr Genet. 2015;61:347-57.

19. Fillinger S, Chaveroche MK, Van Dijck P, de Vries R, Ruijter G, Thevelein J, et al. Trehalose is required for the acquisition of tolerance to a variety of stresses in the filamentous fungus Aspergillus nidulans. Microbiol. 2001;147:1851-62.

20. Jiang J, Liu X, Yin Y, Ma Z. Involvement of a velvet protein FgVeA in the regulation of asexual development, lipid and secondary metabolisms and virulence in Fusarium graminearum. PLoS One. 2011;6:e28291.

21. Thammahong A, Caffrey-Card AK, Dhingra S, Obar JJ, Cramer RA. Aspergillus fumigatus trehalose-regulatory subunit homolog moonlights to mediate cell wall homeostasis through modulation of chitin synthase activity. MBio. 2017;8:e00056-17.

22. Thammahong A, Dhingra S, Bultman KM, Kerkaert JD, Cramer RA. An Ssd1 homolog impacts trehalose and chitin biosynthesis and contributes to virulence in Aspergillus fumigatus. mSphere. 2019;4:e00244-19.

23. Brown AJ, Budge $S$, Kaloriti D, Tillmann A, Jacobsen MD, Yin Z, et al. Stress adaptation in a pathogenic fungus. J Exp Biol. 2014b;217:144-55.

24. Zheng D, Zhang S, Zhou X, Wang C, Xiang P, Zheng Q, et al. The FgHOG1 pathway regulates hyphal growth, stress responses, and plant infection in Fusarium graminearum. PLoS One. 2012;7:e49495.

25. Ding Z, Yang L, Wang G, Guo L, Liu L, Wang J, et al. Fusaric acid is a virulence factor of Fusarium oxysporum $\mathrm{f}$. sp. cubense on banana plantlets. Trop Plant Pathol. 2018:43:297-305.

26. Arras SD, Fraser JA. Chemical inhibitors of non-homologous end joining increase targeted construct integration in Cryptococcus neoformans. PLoS One. 2016;11:e0163049.

27. Ghag SB, Shekhawat UK, Ganapathi TR. Native cell-death genes as candidates for developing wilt resistance in transgenic banana plants. AoB Plants. 2014;6;:plu037.

28. Pfaffl MW, Horgan GW, Dempfle L. Relative expression software tool (RESTC) for group-wise comparison and statistical analysis of relative expression results in real-time PCR. Nucleic Acids Res. 2002;30:e36.

29. Ganapathi TR, Higgs NS, Balint-Kurti PJ, Arntzen CJ, May GD, Van Eck JM. Agrobacterium-mediated transformation of embryogenic cell suspensions of the banana cultivar Rasthali (AAB). Plant Cell Rep. 2001;20:157-62.

30. Ghag SB, Shekhawat UKS, Ganapathi TR. Petunia floral defensins with unique prodomains as novel candidates for development of fusarium wilt resistance in transgenic banana plants. PLoS One. 2012;7:e39557.

31. Rispail N, Di Pietro A. The two-component histidine kinase Fhk1 controls stress adaptation and virulence of Fusarium oxysporum. Mol Plant Pathol. 2010;11:395-407.

32. Pérez-Nadales E, Di Pietro A. The membrane mucin Msb2 regulates invasive growth and plant infection in Fusarium oxysporum. Plant Cell. 2011;23:1171-85.

33. Chandramohan D, Mahadevan A. Detection of fusaric acid in the mycelium and conidia of Fusarium oxysporum f. vasinfectum. Cell Mol Life Sci. 1968;24:427.

\section{Publisher's Note}

Springer Nature remains neutral with regard to jurisdictional claims in published maps and institutional affiliations. 